\title{
Construcción y validación de instrumentos para evaluar prácticas de convivencia escolar en profesionales y padres
}

Construction and validation of instruments to assess school climate practices in school staff and parents

\section{Volumen 18, Número 3 \\ Setiembre-Diciembre}

pp. 1-29

\section{Este número se publica el 1 de setiembre de 2018}

DOI: https://doi.org/10.15517/aie.v18i3.34328

\author{
Verónica López \\ René Valdés Morales
}

Revista indizada en REDALYC, $\underline{\text { SCIELO }}$

Revista distribuida en las bases de datos:

LATINDEX, DOAJ, REDIB, IRESIE, CLASE, DIALNET, SHERPA/ROMEO, QUALIS-CAPES, MIAR

Revista registrada en los directorios:

ULRICH'S, $\underline{\text { REDIE}}, \underline{\text { RINACE}}, \underline{\text { OEI }}$ MAESTROTECA, PREAL, $\underline{\text { CLACSO }}$ 


\title{
Construcción y validación de instrumentos para evaluar prácticas de convivencia escolar en profesionales y padres \\ Construction and validation of instruments to assess school climate practices in school staff and parents
}

\author{
Verónica López ${ }^{1}$ \\ René Valdés Morales ${ }^{2}$
}

\begin{abstract}
Resumen: Los altos niveles de violencia reportados en las instituciones escolares han hecho de la convivencia escolar un tema relevante para las escuelas. No obstante, si bien existen algunos instrumentos disponibles que evalúan clima escolar y percepción de la calidad de la convivencia escolar, pocos evalúan prácticas concretas de convivencia escolar orientadas al mejoramiento escolar, y menos aún desde la perspectiva de profesionales y de la familia. Mediante un abordaje metodológico cuantitativo, en este artículo se exponen los resultados del diseño de las propiedades psicométricas de dos instrumentos que evalúan prácticas de convivencia escolar reportadas; el primero, por padres y/o madres, y el segundo, por profesionales (profesores/as, directivos, profesionales de apoyo y asistentes de la educación). El instrumento fue construido por un equipo de investigación y aplicado al interior de las escuelas durante la jornada escolar. La validación de ambos instrumentos se llevó a cabo con una muestra de 741 profesionales y 1129 padres/madres pertenecientes a 129 escuelas de diferente dependencia escolar, bajo un diseño muestral representativo bietápico y mediante un análisis factorial confirmatorio. Como resultados, ambos instrumentos presentan indicadores de ajuste y fiabilidad aceptables, con ítems con estimación estandarizada sobre .50 y coeficientes de alfa de Cronbach sobre .7. Se discute la importancia de contar con instrumentos que permitan evaluar prácticas concretas de convivencia escolar desde la perspectiva de los profesionales y de los padres/madres.
\end{abstract}

Palabras clave: evaluación; convivencia escolar; educación primaria; prácticas; Chile

\begin{abstract}
The high levels of school violence reported in school institutions have made school climate a relevant issue for schools. However, while there are some instruments available that assess school climate and the perception of the school life quality, few of them assess specific practices of school climate oriented to school improvement, and even less from the perspective of school staff and parents. Through a quantitative methodological approach, in this article we present findings of the construction and psychometric analyses of two instruments that evaluate school climate practices reported by parentsand by school staff (professionals, managers, support professionals and education assistants). The validation of both instruments was carried out with a sample of 741 professionals and 1129 parents, belonging to 129 schools of different administration in Chile, under a two-stage representative sample design. Findings from reliability and confirmatory factor analyses showed suitable adjustment and reliability indicators for both instruments. Items showed a standardized estimate of .50 and coefficients of Cronbach's alpha above .7. We discuss the relevance of having instruments that allow evaluating concrete practices of school climate from the perspective of school staff and parents.
\end{abstract}

Key words: evaluation; school climate; elementary school; practice; Chile

\footnotetext{
1 Profesora de la Pontificia Universidad Católica de Valparaíso, Escuela de Psicología, Viña del Mar, Chile. Doctora en Psicología Dirección electrónica: veronica.lopez@pucv.cl

2 Profesor agregado de la Pontificia Universidad Católica de Valparaíso, Escuela de Pedagogía, Viña del Mar, Chile.Dirección electrónica: revalmorales@gmail.com
}

Articulo recibido: 29 de marzo, 2018

Enviado a corrección: 19 de junio, 2018

Aprobado: 13 de agosto, 2018 


\section{Introducción}

La convivencia escolar ha sido una preocupación tanto de los países anglosajones (Benbenishty, Astor, Roziner y Wrabel, 2016; Cohen y Thapa, 2017; Wang y Degol, 2016) como de los países latinoamericanos (Gairín y Barrera-Corominas, 2014). Desde comienzos de la década del 2000, varios países latinoamericanos han creado leyes y políticas de convivencia escolar. Estos documentos legales han obligado a constituir cuerpos colegiados y/o órganos profesionales al interior de las escuelas para abordar la violencia y la convivencia, otorgándoles atribuciones y haciéndolos responsables de gestionar la convivencia escolar. Tal es el caso de Colombia, cuya Ley $N^{\circ} 16201$ del 2013, crea el "Sistema Nacional de Convivencia Escolar y Formación para el Ejercicio de los Derechos Humanos, la Educación para la Sexualidad y la Prevención y Mitigación de la Violencia Escolar" e instala un comité escolar de convivencia en el colegio y comités a nivel de municipio, distrito, departamento y país.

En Costa Rica, el Ministerio de Educación Pública (MEP) y los marcos normativos referentes a niñez y adolescencia han impulsado una serie de medidas para combatir el bullying al interior de las escuelas; no obstante, el énfasis se ha puesto en la intervención más que en la prevención (Durán Rodríguez, 2016). En Perú, la Ley № 29.719, que promueve la convivencia sin violencia en las instituciones educativas, responsabiliza a los y las profesionales del campo de la psicología de la convivencia en los centros educativos, y crea un consejo educativo institucional por colegio, el cual debe desarrollar un plan de sana convivencia y disciplina escolar.

En Argentina, la Ley №26.892 del año 2013 para la promoción de la convivencia y el abordaje de la conflictividad social en las instituciones educativas, garantiza el derecho a una convivencia pacífica, pero no crea un organismo propio de cada colegio para conducir acciones en convivencia escolar, sino que indica lo que los colegios debiesen enfatizar. En Chile, la Ley $\mathrm{N}^{\circ} 20.536$ Sobre Violencia Escolar del año 2011, cuando se incorpora y modifica la Ley General de Educación N ${ }^{\circ}$ 20.370, crea la figura del encargado/a de convivencia escolar. Además, reconoce de forma explícita que los colegios pueden expulsar a los y a las estudiantes si es que estos/as no han respetado las normas de convivencia escolar, y establece la obligación de los centros escolares de velar por un debido procedimiento en la atención a las denuncias de maltrato escolar, con posibilidad de que el Estado, a través de la Superintendencia de Educación Escolar, sancione monetariamente a las escuelas que no logran demostrar evidencias de un debido proceso. 
Sin embargo, se evidencia una situación paradójica. Por una parte, los países han avanzado en la creación y dictamen de leyes y políticas en materia de convivencia escolar, que exigen a las escuelas a mejorar la convivencia escolar. Sin embargo, se recoge la percepción de que existen pocos lineamientos y conocimientos acerca de cómo realizarlo (Gatica, 2017; Valenzuela, Ahumada, Rubilar, López y Urbina, 2018). Al respecto, la literatura internacional muestra que para mejorar la convivencia escolar es necesario poner énfasis en elementos organizacionales y de gestión, puesto que lo que se requiere es cambiar aquellas prácticas que tienden a ver los problemas de convivencia como problemas individuales y no como problemas organizacionales (López, Carrasco, Morales, Ayala, López y Karmy, 2011).

Por ejemplo, la Organización Mundial de la Salud (OMS) y la literatura sobre prácticas escolares basadas en evidencia (evidence-based practices) muestran que, para prevenir la violencia y mejorar la convivencia escolar, es necesario diseñar e implementar intervenciones a nivel de escuela total (whole school approach) (De Pedro, Pineda, Capp, Moore, Benbenishty y Astor, 2017; Elfrink, Goldberg, Schreurs, Bohlmeijer y Clarke 2017; Thomas y Aggleton, 2016) con tres niveles de intervención (American School Counselor Association, 2003; Dimmit y Robillard, 2014; Kazak et al., 2010; Speece, Case y Molloy, 2003; Wilson, Gottfredson y Najaka, 2001).

El primer nivel (tier 1) correspondería a acciones de promoción o prevención primaria, indicadas para la totalidad de los y las estudiantes, pues buscan mejorar las condiciones pedagógicas del alumnado (ej. buenas clases con adecuado nivel de enseñanza, espacios seguros y agradables para aprender, reglas claras y conocidas). El segundo nivel (tier 2) correspondería a acciones de prevención secundaria, y es indicado para un porcentaje menor de estudiantes (entre el 10 y 20\%) que requieren apoyos específicos, los que se suelen ofrecer de manera grupal bajo la modalidad de talleres (por ejemplo, talleres de reforzamiento pedagógico, talleres de habilidades sociales). Finalmente, el tercer nivel (tier 3) correspondería a acciones de prevención terciaria o intervención individual, y está indicado para un número aún menor de estudiantes (entre el 5 y 10\%) quienes, habiendo pasado por los niveles 1 y 2 , requieren más apoyo específico (por ejemplo, en este nivel se sitúan las derivaciones y la atención individual de estudiantes).

Los modelos de escuela total requieren que el conjunto de las personas que conforman la comunidad escolar, particularmente la población adulta responsable de la escuela directores/as, profesores/as y asistentes - articulen sus acciones de manera coherente y 
consistente. Como vemos, no basta con la voluntad de mejorar, pues muchas veces las voluntades personales se topan con elementos de tipo organizacional que impiden 0 dificultan el mejoramiento. Desde este punto de vista, resulta relevante conocer y evaluar las formas en que las escuelas gestionan la convivencia escolar a través de sus prácticas cotidianas. En la medida en que las escuelas conozcan cuáles son sus prácticas de gestión de la convivencia escolar pueden identificar nudos críticos, tomar conciencia de ellos y generar acciones de reflexión y, posiblemente, de rediseño organizacional.

\subsection{Prácticas de gestión de la convivencia escolar}

A diferencia del concepto de clima escolar, que suele entenderse como la percepción y sensación que una persona tiene a partir de sus experiencias en el sistema escolar (Sandoval Márquez, 2014), o como la calidad y el carácter de una escuela (Cohen, McCabe, Michelli, y Pickeral, 2009), en este estudio el interés está en encontrar modos para evaluar las prácticas de convivencia escolar. Estas se pueden comprender como el conjunto de prácticas de inclusión o de segregación, de participación o de subordinación, de solución pacífica o violenta, de las diferencias entre las personas, y de las adjetivaciones posibles que adquiere la convivencia, en una escuela singular y en un tiempo determinado (Chaparro Caso-López, Caso Niebla, Fierro Evans y Díaz López, 2015; Fierro Evans, 2013). Por lo tanto, y siguiendo la misma diferencia, cuando hablamos de prácticas no nos referimos, a priori, a percepciones, sino a aquellas acciones concretas que se dan en un contexto escolar.

Desde los estudios de la práctica (Gherardi, 2009, 2012; Fardella y Carvajal, 2018), estas se definen como un un patrón de actividades, socialmente reconocido y normalizado que se construye colectivamente (Gherardi, 2009). Esta misma autora señala que las prácticas constituyen un modo de ordenar el flujo de las relaciones organizacionales. Proporcionan un principio de ordenamiento, como la institucionalización de actividades y formas de hacer que se sustentan tanto en las relaciones materiales como sociales. Sin embargo, simultáneamente, este principio ordenador es también temporal e inestable, y por lo tanto es también un principio desorganizador. Se trata de una manera de hacer las cosas donde no solo participan las personas, sino también los dispositivos materiales no-humanos por cuanto operan como agentes en una red de acciones que van construyendo un patrón de actividades (Fardella y Carvajal, 2018; Programa de las Naciones Unidas para el Desarrollo, 2009). 
La literatura internacional documenta cierto tipo de prácticas que tienden a mejorar la convivencia escolar. Un conjunto de ellas refiere ciertas disposiciones organizacionales al cambio (organizational readiness to change) (Helfrich, Li, Sharp y Sales, 2009; Stevens, 2013). La disposición organizacional al cambio, aplicada al ámbito de las escuelas, involucra creencias compartidas de que es posible mejorar. Se trata de una disposición, en tanto involucran un componente motivacional a nivel de la organización, entendido como un nivel de compromiso institucional con "hacer las cosas bien" que va más allá de ciertos compromisos de profesores/as o directivos individuales (Helfrich et al., 2009). En estas escuelas se observan aprendizajes de tipo organizacional (Senge et al., 2000). Esto requiere desarrollar un nivel de confianza entre profesores/as y directivos y asumir colectivamente la responsabilidad por el mejoramiento, en este caso de la convivencia escolar.

Desde esta perspectiva, lo que interesa es conocer cuán preparado o dispuesto se encuentra un centro escolar para el cambio organizacional, en este caso, para la gestión de la convivencia escolar (Carey, Harrity y Dimmitt, 2005; Stevens, 2013). Así, aproximarse a la disposición organizacional al cambio permite trabajar con las instituciones a partir de su potencial de cambio, en vez de solo a través de la reducción de las resistencias (Armenakis, Harris y Feild, 2000). La disposición organizacional al cambio involucra no solo actitudes y creencias de que el cambio es posible, deseable y necesario (Armenakis et al., 2000), sino que, además, comprende comportamientos específicos que demuestran apertura y compromiso con el cambio (Prochaska, Prochaska y Levesque, 2001; Stevens, 2013; Weiner, Amick y Lee, 2008; Tyre, Feuerborn, Beisse, y McCready, 2012).

Aplicando este marco al ámbito de la convivencia escolar (Cohen, Espelage, Twemlow, Berkowitz y Comer, 2015), en Estados Unidos, el National School Climate Center (ver cita en Dary y Pickeral, 2013) desarrolló una herramienta de autoevaluación para medir la disposición organizacional al mejoramiento del clima escolar de las escuelas (School Climate Improvement Readiness Assessment Self-Study Tool). La herramienta consiste en una rúbrica de autoevaluación institucional a nivel de escuela que mide cuán preparada está la institución para apoyar y fomentar una visión compartida; para comprometerse de manera medible y clara con el proceso de mejora del clima escolar; para formar un equipo de liderazgo representativo y democrático; para avanzar hacia una cultura de confianza y resolución colaborativa de problemas; para asegurar disponer de los recursos adecuados para respaldar el proceso de mejoramiento; para celebrar los éxitos y aprender de los 
esfuerzos pasados y actuales; para promover las capacidades reflexivas de sus miembros; y para desarrollar comunidades de aprendizaje profesional.

Por otra parte, la propia literatura sobre mejoramiento escolar, cuya tradición está asentada en los estudios sobre eficacia escolar y mejora escolar (Hallinger y Heck, 2011; Creemers y Kyriakides, 2010) ha evidenciado que la gestión escolar y las prácticas asociadas son elementos claves. Según Elmore y City (2007), el camino a la mejora escolar es difícil, pedregoso y lento, pues involucra un giro desde las acciones individuales hacia prácticas institucionales que logran cambiar las prácticas de la sala de clases. Dentro de estas prácticas, la convivencia escolar - bajo el nombre de clima escolar en el contexto anglosajón - ha comenzado a ser incluido como uno de los elementos que distinguen las escuelas que evidencian mejoras en los aprendizajes y en otros ámbitos distintivos. En Estados Unidos, Wang y Degol (2016) han incorporado la dimensión académica como parte de la definición teórica y operacional de clima escolar, subdividiéndola en liderazgo pedagógico, instrucción/aprendizaje y desarrollo profesional.

En Chile, Bellei, Vanni, Valenzuela y Contreras (2015), basándose en la clasificación de Gray et al. (1999), sobre el desempeño real y potencial de las escuelas, identifican trayectorias de mejoramiento escolar en escuelas chilenas que habían demostrado evidencias cuantitativas de mejoramiento escolar durante una década. Para efectos de este estudio, resulta relevante constatar que dichas trayectorias incorporan prácticas de gestión de la convivencia escolar. En un nivel inicial implicaban como estrategia institucional, tácticas a corto plazo enfocadas a mejorar el rendimiento de los estudiantes en la prueba estandarizada nacional, como instruir para la disciplina y el orden.

Por el contrario, las trayectorias más consolidadas estarían más institucionalizadas e involucrarían esfuerzos por desarrollar capacidades en las personas de la escuela, tales como trabajo colaborativo, liderazgo distribuido y la habilidad para adaptarse a los cambios. En su estudio, las escuelas de nivel de mejoramiento incipiente buscaban producir un ambiente positivo a través de un mayor respeto y confianza entre los y las agentes educativos, y a través del aumento de las instancias de participación de profesores/as y de la familia; en cambio, en las escuelas con trayectoria en vías de institucionalización los/las profesores/as manifestaban sentirse parte de un equipo mayor, con prácticas de colaboración, de inducción a la cultura organizacional y de aprender a aprender cómo mejorar continuamente la convivencia escolar desde una mirada organizacional. 
Si bien existen algunos instrumentos disponibles que evalúan clima escolar y percepción de la calidad de la convivencia escolar (Gálvez Nieto et al., 2014; López, Bilbao, Ascorra, Moyay Morales, 2014) existen pocos instrumentos que evalúan prácticas organizacionales concretas de gestión de la convivencia escolar orientadas a la mejora de la convivencia escolar, y menos aún desde la perspectiva de las personas adultas (profesionales y familia) de la escuela. En este estudio, el objetivo fue construir y validar dos instrumentos de evaluación de prácticas de gestión de la convivencia escolar, considerando las voces de la familia y de profesionales, que permita, mediante la evaluación rigurosa y sistemática, la toma de decisiones desde la gestión formativa. Es importante enfatizar que el instrumento revela acciones concretas que realizan las y los profesionales al interior de las escuelas, alejándose de la evaluación de las percepciones o evaluaciones de calidad que generalmente se evalúan bajo el constructo de clima escolar.

\section{Metodología}

El presente estudio responde a una investigación de metodología cuantitativa (Hernández, Fernández y Baptista, 2010), en la cual se somete a validez y confiabilidad un instrumento que evalúa prácticas de gestión de la convivencia escolar.

\subsection{Participantes}

Participaron padres/madres, docentes, profesionales de apoyo, personal directivo y asistentes de la educación pertenecientes a 129 escuelas representativas de tres regiones de Chile. La elección de las escuelas siguió un diseño muestral estratificado bietápico (nivel de confianza al 95\% y margen de error del 10\%). En la primera etapa, se seleccionaron las tres comunas del país que tenían alto nivel de denuncias por maltrato escolar, según información oficial de la Superintendencia de Educación Escolar. En la segunda etapa, se seleccionaron aleatoriamente escuelas representativas de la proporción de escuelas municipales, particular-subvencionadas y privadas de cada región. En todas las escuelas se invitó a participar a los padres y/o las madres de los estudiantes de octavo año básico ( $n=1129)$, los/as profesores/as que enseñaban a esos estudiantes $(n=376)$, personal directivo $(n=152)$, profesionales de apoyo psicosocial $(n=66)$ compuesto por psicólogos/as, trabajadores/as sociales y fonoaudiólogos/as con permanencia en la escuela, y asistentes de aula, personal de aseo y administrativos, también llamados paradocentes ( $n=147)$. Así, en 
total participaron 1870 adultos de las escuelas participantes (ver Tabla 1). A cada grupo de participantes (padres/madres y profesionales) se les aplicó un instrumento diferente.

Tabla 1

Características demográficas de la muestra por sexo y en tres regiones de Chile, 2016 -2017. (Valores nominales y relativos)

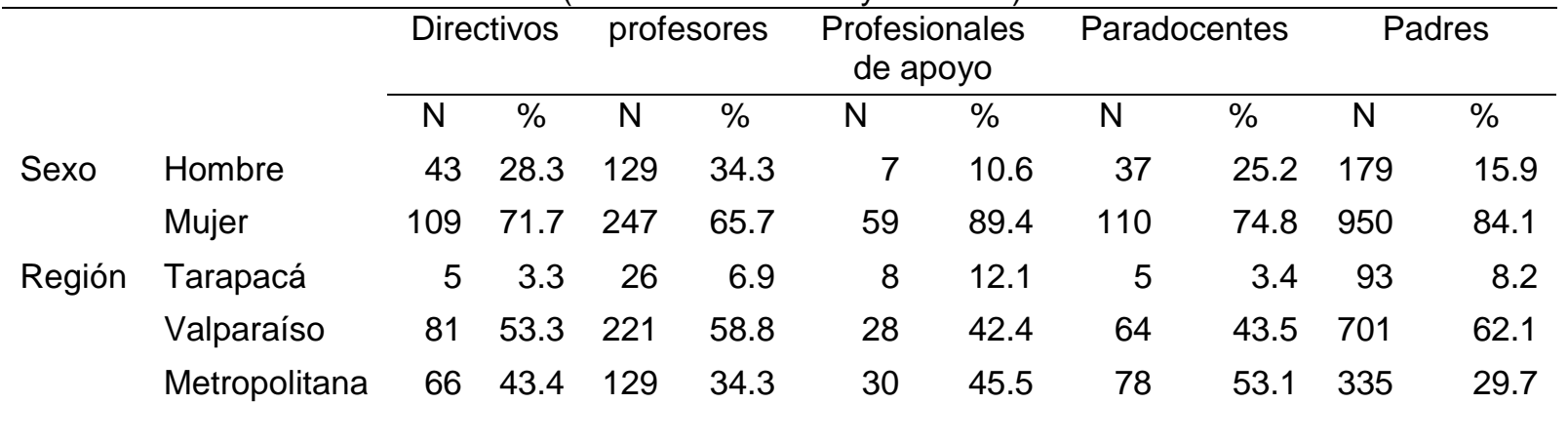

Fuente: Elaboración propia, 2017.

\subsection{Instrumento}

Prácticas de convivencia escolar. La construcción de los instrumentos se diseñó tomando como referencia teórica-conceptual las prácticas de convivencia escolar, las prácticas orientadas a crear culturas escolares inclusivas (Booth y Ainscow, 2015) y la disposición organizacional al cambio (Helfrich et al., 2009; Stevens, 2013), aplicado al ámbito del clima y convivencia escolar. Las dimensiones y los ítems se construyeron tomando en cuenta instrumentos y/o propuestas evaluativas que tuvieran vínculo con las prácticas de convivencia escolar.

Las fuentes bibliográficas fueron las siguientes:

a) Instrumento de evaluación basado en indicadores de convivencia escolar democrática, inclusiva y pacífica (Chaparro et al., 2015; Valdés, López y Chaparro, 2018, en prensa): este instrumento reporta las prácticas de convivencia en las escuelas desde el punto de vista democrático, inclusivo y pacífico;

b) Instrumento de evaluación de la disposición organizativa para el cambio (Helfrich et al., 2009): este instrumento agrupa indicadores alusivos a la disposición de la organización para evaluar el cambio a través de tres escalas: (i) evidencia, que está destinado a cómo el equipo se ve a sí mismo, y los trabajadores ven a sus colegas; (ii) contexto, que evalúa la cultura organizacional; y (iii) facilitación, que evalúa las características de la gestión del liderazgo; 
c) Instrumento para evaluar la disposición al cambio (Stevens, 2013): este instrumento hace alusión a la importancia de las creencias, actitudes e intenciones hacia el cambio, tales como la apertura al cambio, el apoyo de dirección y la disminución de las resistencias;

d) Rúbrica de evaluación de clima escolar (National School Climate Center, 2014): es una herramienta que está diseñada para la autoevaluación por parte de la escuela respecto a su disposición organizacional para el mejoramiento del clima escolar en los dominios: (i) el apoyo y fomento de una visión compartida de escuela, (ii) el compromiso medible y delineado para el proceso de mejora de clima escolar, (iii) la formación de un equipo de liderazgo representativo, (iv) una cultura de confianza y de resolución colaborativa de problemas, (v) recursos para apoyar los procesos de mejora, (vi) la disposición a celebrar los éxitos y los esfuerzos de todos, (vii) la promoción de capacidades reflexivas, y (viii) el desarrollo de comunidades de aprendizaje.

Al mismo tiempo, el equipo a cargo diseñó nuevas preguntas (o ítems emergentes) para las dimensiones de prácticas punitivas, prácticas de prevención de los problemas de convivencia y prácticas de promoción de la buena conciencia. En diversas sesiones de trabajo, cada integrante del grupo propuso indicadores en función de las dimensiones antes mencionadas. El instrumento para profesionales quedó conformado por 12 dimensiones teóricas para profesionales (78 ítems). Las mismas 12 dimensiones teóricas fueron consideradas para el instrumento para la familia, pero con menos ítems (73 ítems). Esta diferencia se explica por la adaptación léxica y semántica al instrumento de padres y madres y por la imposibilidad de estos mismos de responder a asuntos internos de la escuela debido a probables faltas de conocimiento. Por esta razón, en este artículo se consideran y analizan los dos instrumentos por separado (uno para la familia y otro para profesionales de la escuela).

En ambos instrumentos la consigna general solicitaba contestar un cuestionario sobre la convivencia en el colegio. Se señalaba que se presentarían algunas acciones que podrían suceder en su colegio, sobre las que la persona debía expresar la frecuencia en que ocurrían en su opinión. Las opciones de respuestas eran cinco (1=nunca, 2=algunas veces, $3=$ regularmente, $4=$ casi siempre y $5=$ siempre). Se utilizan opciones impares de respuesta, pues estas ofrecen mayores facilidades para quien responde y también son consideradas de mayor preferencia entre las personas (Pérez, Rodríguez, Romero, Ruvalcaba y Lozano, 
2002). A continuación, se presentan las definiciones teóricas y operativas de las 11 dimensiones consideradas para ambos instrumentos:

Cultura inclusiva (8 ítems para profesionales y 7 ítems para la familia): refiere a la creación de una comunidad segura, acogedora, colaboradora y estimulante en la que cada uno es valorado, como el fundamento primordial para que todo el alumnado tenga los mayores niveles de logro (Booth y Ainscow, 2015). Ejemplo: Existe un ambiente familiar.

Prácticas inclusivas (6 ítems para ambas versiones): se refiere a que las prácticas educativas reflejan la cultura y las políticas inclusivas de la escuela. Tiene que ver con asegurar que las actividades en el aula y las actividades extraescolares promuevan la participación de todo el alumnado y tengan en cuenta el conocimiento y la experiencia adquiridos por los estudiantes fuera de la escuela (Booth y Ainscow, 2015). Ejemplo: Existen estrategias efectivas para potenciar a los estudiantes con intereses diversos.

Formación continua (8 ítems para profesionales y 6 ítems para la familia): refiere la presencia intencionada de una cultura de mejoramiento continuo por parte de los profesionales de la escuela. Ejemplo: Al equipo directivo le interesa el desarrollo y la formación continua de los profesores.

Orientación al trabajo en equipo (5 ítems para ambas versiones): hace referencia a la presencia de prácticas y objetivos comunes dentro de la comunidad escolar. Ejemplo: Todos tenemos claros nuestros objetivos como miembros de esta comunidad escolar.

Normas compartidas (5 ítems para ambas versiones): trata acerca de la presencia de un reglamento escolar compartido por todos, tanto en diseño como en ejecución. Ejemplo: Las normas son claras y conocidas por todos/as.

Participación democrática (11 ítems para ambas versiones): se refiere a la motivación de los distintos miembros de la comunidad escolar y al nivel de consideración en la toma de decisiones de la escuela. Ejemplo: Los estudiantes están motivados y participan activamente en la vida de la escuela.

Prácticas punitivas (6 ítems para ambas versiones): consiste en la aplicación de un castigo como medida principal de intervención, ya sea la apertura de un expediente disciplinario o una expulsión del aula o del centro por el tiempo que se considere oportuno, según la gravedad de los hechos (Villaoslada y Torrego, 2004). Ejemplo: Se castiga a los estudiantes que presentan problemas de conducta.

Actitudes de disposición al cambio (4 ítems para ambas versiones): se refiere a la importancia de las creencias, actitudes e intenciones para implementar cambios en una 
institución. Involucra disposición y apertura al cambio, percepción de que la dirección de la escuela apoya el cambio, así como de un bajo nivel de resistencias al cambio (Stevens, 2013). Ejemplo: Creemos que es necesario incorporar cambios para mejorar la convivencia escolar.

Disposición organizacional (6 ítems para profesionales y 5 ítems para la familia): consiste en cómo el equipo se ve a sí mismo y a sus colegas, a la cultura organizacional y a las características de la gestión (Helfrich et al., 2009). Ejemplo: Los profesores y profesionales de apoyo conforman un equipo de trabajo cohesionado.

Cultura de mejoramiento de la convivencia (8 ítems para profesionales y 7 ítems para la familia): presencia intencionada de una cultura de mejoramiento de la convivencia escolar en la comunidad escolar. Ejemplo: El equipo directivo, profesores y profesionales no docentes comparten la misma visión sobre convivencia escolar.

Prevención de los problemas de convivencia (6 ítems para ambas versiones): gestión de la escuela hacia la identificación de posibles barreras, prácticas o conductas que afecten la convivencia escolar. Ejemplo: Se adoptan medidas preventivas para evitar los problemas de convivencia.

Promoción de la buena convivencia (5 ítems para ambas versiones): da cuenta de una gestión de tipo formativa de la escuela orientada a la promoción de prácticas que mejoren la convivencia escolar. Ejemplo: Se promueve la resolución pacífica de conflictos.

Denuncias por maltrato escolar y discriminación. Adicionalmente, se consideró como medida de validez de criterio el nivel de denuncias por maltrato escolar $(M=0.30, D E=0.7$, Rango $=0$ a 4) y discriminación recibidas en la Superintendencia de Educación Escolar de las escuelas participantes del estudio $(M=0.15 \mathrm{DE}=0.4$, Rango $=0$ a 2$)$.

\subsection{Procedimiento y análisis de datos}

En primer lugar, para analizar la validez de contenido, se realizó un análisis léxico y de pertinencia semántica de los ítems de ambos instrumentos con la finalidad de resguardar la precisión lingüística de los reactivos a la realidad chilena. Este análisis estuvo destinado a todas las dimensiones y fue el equipo de investigación a cargo quien construyó y aplicó los dos instrumentos al interior de las escuelas. Como existen ítems que se aplican tanto a profesionales como a padres y madres, se resguardó que estos últimos comprendieran frases como la siguiente: "Los profesores monitorean constantemente el aprendizaje de los estudiantes", cambiando la palabra "monitorean" por "supervisan". Además, en esta 
investigación se consideraron ítems de la literatura anglosajona, por lo tanto, estos reactivos merecen un trato semántico para la aplicación en el contexto chileno. Por ejemplo, cambiamos las frases "padres de familia" por "padres y apoderados" o palabras como "Platicar" o "salón de clases", por "dialogar" y "sala de clases", respectivamente, que son palabras utilizadas en nuestro contexto.

Posteriormente, el equipo a cargo de la aplicación de los instrumentos redactó y aplicó los consentimientos informados destinados a la familia, profesionales de apoyo, profesores/as y directivos. Estos firmaron cada protocolo ético antes de contestar el cuestionario. Después de la autorización de los colegios y la firma de consentimientos informados por parte de los participantes, el instrumento fue aplicado en forma individual en las mismas dependencias de los establecimientos. Para estudiar la validez de constructo se realizó un análisis factorial confirmatorio (AFC), realizando una estimación de máxima verosimilutud mediante el programa Amos 21.0. Se realizaron modelos de primer orden para cada una de las dimensiones de los dos instrumentos, explorando los índices de ajuste, cargas factoriales y coeficientes estimados.

Seguidamente, se probó un modelo de segundo orden para cada instrumento de acuerdo con la pertinencia de las dimensiones a un factor mayor de convivencia escolar. Para analizar la confiabilidad del instrumento, se calculó el índice Alpha de Cronbach para cada una de las dimensiones resultantes de los AFC anteriores. Finalmente, para analizar la validez de criterio se realizó un análisis de correlaciones bivariadas con el nivel de denuncias por maltrato y discriminación recibidas en la Superintendencia de Educación al finalizar el año escolar.

\section{Resultados}

Los resultados del AFC indicaron, en general, índices de ajuste aceptables en ambos instrumentos, conforme a las dimensiones teóricamente esperadas (ver Tabla 2), teniendo como referencia los siguientes valores: CFI mayor a .90, RMSEA menor a.05, GFI menor a .95, TLI igual o mayor a .95, SRMR menor a .08 (Byrne, 2010). Además, se consideró un nivel de significancia igual o menor 0.05 para la prueba de hipótesis. Para este caso, no se consideró el chi cuadrado como medida de ajuste confiable, pues este estadístico es sensible al tamaño muestral, siendo recomendado su uso para muestras entre 100 y 200 casos (Martínez, 2004). 
El análisis de las estimaciones no estandarizadas y estandarizadas de cada ítem respecto al factor subyacente indicó que, salvo en algunas excepciones, los ítems tenían una estimación estandarizada sobre .50 respecto a la dimensión (ver Tabla 3). Las excepciones están señalizadas en negritas en la Tabla 3, y el ítem respectivo puede ser identificado en el Anexo 1.

Eliminados los ítems con estimación estandarizada bajo .50 (específicamente, ítems 5 y 6 para la dimensión prácticas punitivas-profesionales, e ítem 6 para la dimensión prácticas punitivas-familia; ítem 6 para prevención de problemas de convivencia-profesionales e ítem 5 para promoción de buena convivencia escolar-profesionales; ítem 1 para la dimensión de actitudes de disposición al cambio), los indicadores de ajuste del AFC mejoraron para todas las dimensiones de profesionales (prácticas punitivas: $\mathrm{CFI}=0.98, \mathrm{RMSEA}=0.08, \mathrm{GFI}=0.99$, $\mathrm{TLI}=0.94, \mathrm{SRMR}=0.24$; prevención de los problemas de convivencia: $\mathrm{CFI}=0.96, \mathrm{RMSEA}=$ 0.14, $\mathrm{GFI}=0.96, \mathrm{TLI}=0.93, \mathrm{SRMR}=0.03$; promoción de la buena convivencia: $\mathrm{CFI}=0.91$, $\mathrm{RMSEA}=0.32, \mathrm{GFI}=0.90, \mathrm{TLI}=0.75, \mathrm{SRMR}=0.05)$.

Se encontraron hallazgos similares para las dimensiones del instrumento de padres (prácticas punitivas: $\mathrm{CFI}=0.99, \mathrm{RMSEA}=0.64, \mathrm{GFI}=0.99, \mathrm{TLI}=0.98, \mathrm{SRMR}=0.01$ ). No obstante, para el caso de la dimensión actitudes de disposición al cambio, los indicadores de ajuste no mejoraron ni para el instrumento de padres/madresni para profesionales. A pesar de esto, se decidió considerar igualmente esta dimensión por la relevancia teórica que ofrece para ambos instrumentos.

Los resultados antes mencionados proponen la eliminación de los ítems señalados en negrita en la Tabla 3, especialmente para el caso de las dimensiones prácticas punitivas, prevención de los problemas de convivencia y promoción de la buena convivencia en el caso de profesionales (Tabla 3). 
Tabla 2

Índices de ajustes del análisis factorial confirmatorio para profesionales y familias

\begin{tabular}{|c|c|c|c|c|c|c|c|c|c|c|}
\hline & \multicolumn{5}{|c|}{ Profesionales } & \multicolumn{5}{|c|}{ Padres } \\
\hline & CFI & RMSEA & GFI & TLI & SRMR & CFI & RMSEA & GFI & TLI & SRMR \\
\hline Cultura inclusiva & 0.92 & 0.11 & 0.92 & 0.90 & 0.04 & 0.97 & 0.11 & 0.97 & 0.96 & 0.02 \\
\hline $\begin{array}{l}\text { Prácticas } \\
\text { inclusivas }\end{array}$ & 0.93 & 0.14 & 0.93 & 0.88 & 0.04 & 0.98 & 0.07 & 0.97 & 0.97 & 0.02 \\
\hline $\begin{array}{l}\text { Formación } \\
\text { continua }\end{array}$ & 0.91 & 0.15 & 0.89 & 0.88 & 0.04 & 0.97 & 0.11 & 0.96 & 0.95 & 0.02 \\
\hline $\begin{array}{l}\text { Orientación } \\
\text { trabajo } \\
\text { equipo en }\end{array}$ & 0.87 & 0.30 & 0.85 & 0.75 & 0.07 & 0.97 & 0.11 & 0.96 & 0.86 & 0.04 \\
\hline Normas & 0.95 & 0.16 & 0.94 & 0.90 & 0.03 & 0.96 & 0.14 & 0.95 & 0.93 & 0.03 \\
\hline $\begin{array}{l}\text { Participación } \\
\text { democrática }\end{array}$ & 0.76 & 0.19 & 0.74 & 0.70 & 0.08 & 0.84 & 0.16 & 0.81 & 0.80 & 0.06 \\
\hline $\begin{array}{l}\text { Prácticas } \\
\text { punitivas }\end{array}$ & 0.94 & 0.08 & 0.97 & 0.91 & 0.03 & 0.95 & 0.10 & 0.96 & 0.92 & 0.04 \\
\hline $\begin{array}{ll}\text { Actitudes } & \text { de } \\
\text { disposición } & \text { de } \\
\text { cambio } & \end{array}$ & 0.96 & 0.16 & 0.97 & 0.90 & 0.04 & 0.96 & 0.16 & 0.97 & 0.89 & 0.04 \\
\hline $\begin{array}{l}\text { Disposición } \\
\text { organizacional } \\
\text { cambio }\end{array}$ & 0.92 & 0.17 & 0.90 & 0.86 & 0.04 & 0.96 & 0.16 & 0.94 & 0.92 & 0.03 \\
\hline $\begin{array}{l}\text { Cultura de } \\
\text { mejoramiento } \\
\text { de la } \\
\text { convivencia }\end{array}$ & 0.96 & 0.09 & 0.95 & 0.95 & 0.02 & 0.97 & 0.09 & 0.96 & 0.96 & 0.02 \\
\hline $\begin{array}{l}\text { Prevención de } \\
\text { los problemas } \\
\text { de convivencia }\end{array}$ & 0.96 & 0.12 & 0.95 & 0.94 & 0.03 & 0.96 & 0.10 & 0.96 & 0.94 & 0.03 \\
\hline $\begin{array}{l}\text { Promoción de la } \\
\text { buena } \\
\text { convivencia }\end{array}$ & 0.91 & 0.20 & 0.91 & 0.83 & 0.05 & 0.96 & 0.10 & 0.96 & 0.78 & 0.05 \\
\hline
\end{tabular}

Fuente: Elaboración propia, 2017.

Tabla 3

Cargas factoriales no estandarizadas (desviaciones estándares) y estandarizadas (desviaciones estándares) para las dimensiones de prácticas de convivencia escolar de profesionales $(n=741)$ y padres y/o madres $(n=1129)$

\begin{tabular}{lcccc}
\hline & \multicolumn{2}{c}{ Profesionales } & \multicolumn{2}{c}{ Padres } \\
\cline { 2 - 5 } & $\begin{array}{c}\text { No estandarizado } \\
\text { (D.E) }\end{array}$ & $\begin{array}{c}\text { Estandarizado } \\
\text { (D.E) }\end{array}$ & $\begin{array}{c}\text { No estandarizado } \\
\text { (D.E) }\end{array}$ & $\begin{array}{c}\text { Estandarizado } \\
\text { (D.E) }\end{array}$ \\
\cline { 2 - 5 } Íltura inclusiva & 1.00 & .73 & 1.00 & .74 \\
Ítem 2 & $.83(.04)$ & .67 & $1.00(.03)$ & .78 \\
Ítem 3 & $.98(.06)$ & .61 & -- & -- \\
Ítem 4 & $.93(.05)$ & .71 & $1.00(.03)$ & .79 \\
Ítem 5 & $.74(.03)$ & .73 & $.95(.03)$ & .79 \\
Ítem 6 & $.81(.04)$ & .69 & $.96(.03)$ & .75 \\
Ítem 7 & $.82(.04)$ & .76 & $.96(.03)$ & .80 \\
Ítem 8 & $1.16(.05)$ & .76 & $1.00(.04)$ & .73 \\
\hline
\end{tabular}




\begin{tabular}{|c|c|c|c|c|}
\hline \multicolumn{5}{|c|}{ Prácticas inclusivas } \\
\hline Ítem 1 & 1.00 & .75 & 1.00 & .73 \\
\hline Ítem 2 & $.90(.04)$ & .80 & $1.09(.04)$ & .81 \\
\hline Ítem 3 & $.98(.04)$ & .80 & $1.14(.04)$ & .83 \\
\hline Ítem 4 & $1.03(.04)$ & .81 & $1.19(.04)$ & .83 \\
\hline Ítem 5 & $.82(.04)$ & .62 & $.92(.04)$ & .64 \\
\hline Ítem 6 & $.72(.04)$ & .62 & $.98(.03)$ & .77 \\
\hline \multicolumn{5}{|c|}{ Formación continua } \\
\hline Ítem 1 & 1.00 & .83 & 1.00 & .81 \\
\hline Ítem 2 & $1.08(.03)$ & .83 & $1.12(.03)$ & .82 \\
\hline Ítem 3 & $1.08(.03)$ & .87 & $1.07(.03)$ & .83 \\
\hline Ítem 4 & $.83(.03)$ & .76 & --- & -- \\
\hline Ítem 5 & $.82(.03)$ & .75 & --- & -- \\
\hline Ítem 6 & $1.04(.03)$ & .84 & $1.01(.03)$ & .81 \\
\hline Ítem 7 & $.92(.04)$ & .69 & $1.01(.03)$ & .74 \\
\hline Ítem 8 & $.80(.03)$ & .69 & $.94(.03)$ & .76 \\
\hline \multicolumn{5}{|c|}{ Orientación al trabajo en equipo } \\
\hline Ítem 1 & 1.00 & .69 & 1.00 & .72 \\
\hline Ítem 2 & $1.06(.05)$ & .74 & $1.03(.04)$ & .75 \\
\hline Ítem 3 & $1.34(.05)$ & .90 & $1.22(.04)$ & .89 \\
\hline Ítem 4 & $1.35(.06)$ & .91 & $1.17(.04)$ & .87 \\
\hline Ítem 5 & $1.25(.06)$ & .75 & $1.14(.04)$ & .76 \\
\hline \multicolumn{5}{|l|}{ Normas } \\
\hline Ítem 1 & 1.00 & .76 & 1.00 & .66 \\
\hline Ítem 2 & $1.08(.04)$ & .80 & $1.29(.05)$ & .74 \\
\hline Ítem 3 & $1.03(.04)$ & .81 & $1.39(.05)$ & .86 \\
\hline Ítem 4 & $1.04(.04)$ & .77 & $1.44(.05)$ & .86 \\
\hline Ítem 5 & $.92(.04)$ & .79 & $1.38(.05)$ & .87 \\
\hline \multicolumn{5}{|c|}{ Participación democrática } \\
\hline Ítem 1 & 1.00 & .66 & 1.00 & .72 \\
\hline Ítem 2 & $.81(.05)$ & .64 & $.98(.04)$ & .68 \\
\hline Ítem 3 & $1.11(.05)$ & .80 & $1.15(.04)$ & .79 \\
\hline Ítem 4 & $1.13(.05)$ & .81 & $1.20(.04)$ & .82 \\
\hline Ítem 5 & $1.23(.06)$ & .80 & $1.25(.04)$ & .84 \\
\hline Ítem 6 & $1.32(.06)$ & .82 & $1.23(.04)$ & .83 \\
\hline Ítem 7 & $1.13(.07)$ & .58 & $1.18(.05)$ & .65 \\
\hline Ítem 8 & $.95(.06)$ & .57 & $1.04(.04)$ & .65 \\
\hline Ítem 9 & $1.18(.07)$ & .67 & $1.22(.04)$ & .77 \\
\hline Ítem 10 & $1.33(0.8)$ & .65 & $1.25(.05)$ & .68 \\
\hline Ítem 11 & $1.26(0.8)$ & .61 & $1.24(.05)$ & .67 \\
\hline \multicolumn{5}{|c|}{ Prácticas punitivas } \\
\hline Ítem 1 & 1.00 & .57 & 1.00 & .78 \\
\hline
\end{tabular}




\begin{tabular}{|c|c|c|c|c|}
\hline Ítem 2 & $1.09(.08)$ & .69 & $1.10(.03)$ & .84 \\
\hline Ítem 3 & $1.23(.08)$ & .79 & $.99(.03)$ & .78 \\
\hline Ítem 4 & $.82(.07)$ & .56 & $.70(.03)$ & .54 \\
\hline Ítem 5 & $.69(0.6)$ & .48 & $.65(.04)$ & .47 \\
\hline Ítem 6 & $.64(.07)$ & .40 & $.71(.03)$ & .62 \\
\hline \multicolumn{5}{|c|}{$\begin{array}{l}\text { Actitudes de disposición al } \\
\text { cambio }\end{array}$} \\
\hline Ítem 2 & $6.48(2.23)$ & .81 & $2.62(.29)$ & .73 \\
\hline Ítem 3 & 6.95 (2.39) & .88 & $3.25(.35)$ & .88 \\
\hline Ítem 4 & $6.79(2.34)$ & .83 & $3.04(.33)$ & .84 \\
\hline \multicolumn{5}{|c|}{ Disposición organizacional } \\
\hline Ítem 1 & 1.00 & .82 & 1.00 & .85 \\
\hline Ítem 2 & $.91(.03)$ & .80 & $.96(.02)$ & .86 \\
\hline Ítem 3 & $.78(.03)$ & .75 & $.87(.02)$ & .82 \\
\hline Ítem 4 & $.94(.03)$ & .80 & $.88(.02)$ & .82 \\
\hline Ítem 5 & $.89(.03)$ & .78 & $.89(.02)$ & .76 \\
\hline Ítem 6 & $.92(.04)$ & .71 & --- & -- \\
\hline \multicolumn{5}{|c|}{ Cultura de mejoramiento de la convivencia } \\
\hline Ítem 1 & 1.00 & .79 & --- & -- \\
\hline Ítem 2 & $1.01(.04)$ & .83 & 1.00 & .84 \\
\hline Ítem 3 & $1.12(.04)$ & .80 & $1.08(.03)$ & .84 \\
\hline Ítem 4 & $1.05(.04)$ & .76 & $1.02(.02)$ & .87 \\
\hline Ítem 5 & $.91(.04)$ & .71 & $.95(.02)$ & .83 \\
\hline Ítem 6 & $1.01(.05)$ & .68 & $.89(.03)$ & .73 \\
\hline Ítem 7 & $.93(.04)$ & .76 & $.93(.02)$ & .82 \\
\hline Ítem 8 & $1.02(.04)$ & .75 & $.96(.02)$ & .84 \\
\hline \multicolumn{5}{|c|}{ Prevención de los problemas de convivencia } \\
\hline Ítem 1 & 1.00 & .70 & 1.00 & .75 \\
\hline Ítem 2 & $1.16(.05)$ & .83 & $1.17(.04)$ & .82 \\
\hline Ítem 3 & $1.25(.05)$ & .89 & $1.22(.04)$ & .88 \\
\hline Ítem 4 & $1.17(.05)$ & .88 & $1.25(.04)$ & .88 \\
\hline Ítem 5 & $.86(.05)$ & .64 & $.97(.03)$ & .72 \\
\hline Ítem 6 & $.29(.04)$ & .24 & $.79(.03)$ & 62 \\
\hline \multicolumn{5}{|c|}{ Promoción de la buena convivencia } \\
\hline Ítem 1 & 1.00 & .87 & 1.00 & .84 \\
\hline Ítem 2 & $1.05(.03)$ & .90 & $1.01(.02)$ & .87 \\
\hline Ítem 3 & $.96(.03)$ & .78 & $.91(.03)$ & .74 \\
\hline Ítem 4 & $.97(.04)$ & .70 & $.98(.03)$ & .75 \\
\hline Ítem 5 & $.33(.05)$ & .24 & $.85(.03)$ & .66 \\
\hline
\end{tabular}

Nota: Todas las cargas son significativas a nivel $p<0.001$

Fuente: Elaboración propia, 2017. 
Con base en estos resultados, y tras eliminar los ítems anteriores, se presentan en la Tabla 4 los estadísticos descriptivos por dimensión, Alpha de Cronbach y percentiles. Según estos datos, los coeficientes indicaron un nivel de consistencia interna aceptable (>.7), buenos (>.8) y excelentes (>.9). Se exponen los percentiles para facilitar el uso de estas dimensiones como formas de evaluación y autoevaluación de las prácticas de convivencia escolar.

Por último, se probó un modelo de segundo orden considerando las 12 dimensiones. Los valores para el instrumento de profesionales mostraron índices de ajuste plausibles, pero no excelentes para el instrumento de profesionales $(\mathrm{CFI}=0.83, \mathrm{RMSEA}=0.06, \mathrm{GFI}=0.70$, $\mathrm{TLI}=0.83, \mathrm{SRMR}=0.05)$ así como para el de padres/madres $(\mathrm{CFI}=0.88, \mathrm{RMSEA}=0.05$, $\mathrm{GFI}=0.78, \mathrm{TLI}=0.88, \mathrm{SRMR}=0.04)$. En ambos instrumentos, las cargas factoriales de las dimensiones, con respecto a su relación con el factor de segundo orden, fueron mayores a 0.80 para todas las dimensiones, menos para la de prácticas punitivas para profesionales (0.02 ) y para padres/madres (0.55), lo cual es teóricamente coherente. Por lo tanto, se probó un segundo modelo de segundo orden eliminando esta dimensión. Los valores de ajuste se mantuvieron tanto para profesionales $(\mathrm{CFI}=0.83, \mathrm{RMSEA}=0.06, \mathrm{GFI}=0.70, \mathrm{TLI}=0.83$, $\mathrm{SRMR}=0.05)$ como para padres/madres $(\mathrm{CFI}=0.83, \mathrm{RMSEA}=0.06, \mathrm{GFI}=0.70, \mathrm{TLI}=0.83$, $\mathrm{SRMR}=0.05)$. Consecuentemente, se recomienda el uso del instrumento según las dimensiones de interés. 
Tabla 4

Estadísticos descriptivos por dimensión y tipo de participantes

\begin{tabular}{|c|c|c|c|c|c|c|c|c|c|c|c|c|}
\hline \multirow[t]{2}{*}{ Dimensiones } & \multicolumn{6}{|c|}{$\begin{array}{c}\text { Profesionales } \\
(741)\end{array}$} & \multicolumn{6}{|c|}{$\begin{array}{c}\text { Padres } \\
(1129)\end{array}$} \\
\hline & $\alpha$ & $M$ & DT & P25 & P50 & P75 & $\alpha$ & $M$ & DT & P25 & P50 & P75 \\
\hline $\begin{array}{l}\text { Cultura } \\
\text { inclusiva }\end{array}$ & .88 & 3.86 & .75 & 3.3 & 4.0 & 4.5 & .91 & 3.87 & .86 & 3.2 & 4.0 & 4.5 \\
\hline $\begin{array}{l}\text { Prácticas } \\
\text { inclusivas }\end{array}$ & .87 & 3.87 & .77 & 3.3 & 4.0 & 4.5 & .89 & 3.71 & .94 & 3.0 & 3.8 & 4.5 \\
\hline $\begin{array}{l}\text { Formación } \\
\text { continua }\end{array}$ & .92 & 3.68 & .95 & 3.0 & 3.8 & 4.5 & .91 & 3.75 & .93 & 3.1 & 3.8 & 4.5 \\
\hline $\begin{array}{l}\text { Orientación } \\
\text { al trabajo en } \\
\text { equipo }\end{array}$ & .90 & 3.81 & .89 & 3.2 & 4.0 & 4.6 & .90 & 3.95 & .88 & 3.4 & 4.0 & 4.8 \\
\hline $\begin{array}{l}\text { Normas } \\
\text { compartidas }\end{array}$ & .89 & 3.97 & .84 & 3.4 & 4.0 & 4.6 & .90 & 3.90 & .91 & 3.3 & 4.0 & 4.6 \\
\hline $\begin{array}{l}\text { Participación } \\
\text { democrática }\end{array}$ & .90 & 3.62 & .79 & 3.0 & 3.6 & 4.2 & .90 & 3.57 & .89 & 2.9 & 3.7 & 4.2 \\
\hline $\begin{array}{l}\text { Prácticas } \\
\text { punitivas }\end{array}$ & .75 & 2.61 & .89 & 2.0 & 2.5 & 3.0 & .83 & 3.48 & .97 & 2.8 & 3.6 & 4.2 \\
\hline $\begin{array}{l}\text { Actitudes de } \\
\text { disposición } \\
\text { al cambio }\end{array}$ & .72 & 4.0 & .79 & 3.5 & 4.0 & 4.7 & .77 & 3.7 & .87 & 3.2 & 3.9 & 4.4 \\
\hline $\begin{array}{l}\text { Disposición } \\
\text { organizacion } \\
\text { al cambio }\end{array}$ & .90 & 3.95 & .83 & 3.3 & 4.1 & 4.6 & .91 & 4.03 & .85 & 3.6 & 4.2 & 4.8 \\
\hline $\begin{array}{l}\text { Cultura de } \\
\text { mejoramient }\end{array}$ & .91 & 3.94 & .85 & 3.2 & 4.1 & 4.6 & .93 & 3.87 & .93 & 3.2 & 4.0 & 4.7 \\
\hline $\begin{array}{l}\text { o de la } \\
\text { convivencia }\end{array}$ & & & & & & & & & & & & \\
\hline $\begin{array}{l}\text { Prevención } \\
\text { de los } \\
\text { problemas } \\
\text { de } \\
\text { convivencia }\end{array}$ & .85 & 4.01 & .87 & 3.4 & 4.2 & 4.8 & .90 & 3.80 & .93 & 3.1 & 4.0 & 4.5 \\
\hline $\begin{array}{l}\text { Promoción } \\
\text { de la buena } \\
\text { convivencia }\end{array}$ & .81 & 4.21 & .85 & 3.7 & 4.5 & 5.0 & .88 & 3.91 & .89 & 3.4 & 4.0 & 4.6 \\
\hline
\end{tabular}

Finalmente, y con la finalidad de contrastar la hipótesis de validez de criterio, se realizó un análisis de correlaciones bivariadas con el nivel de denuncias por maltrato y discriminación de los establecimientos educacionales participantes recibidos el mismo año en la Superintendencia de Educación Escolar. Dado que la Superintendencia recibe denuncias a nivel de establecimiento, para este análisis, se creó una base de datos agregada por establecimiento. Luego, se creó una variable de "prácticas de convivencia inclusiva" considerando las 11 dimensiones con altas cargas factoriales en el AFC de 
segundo orden, y se consideró la dimensión de "prácticas punitivas" como otra variable independiente. Los resultados indican que, para el caso de los profesionales, no se observaron correlaciones significativas entre el nivel de denuncias y las prácticas inclusivas y/o punitivas. En el caso de los padres/madres, sí se observaron correlaciones negativas significativas entre las prácticas de convivencia inclusiva y el total de denuncias por maltrato escolar y discriminación y el total de denuncias por discriminación (ver Tabla 5).

Tabla 5

Correlación entre las prácticas de convivencia escolar y nivel de denuncias por maltrato escolar y discriminación

\begin{tabular}{llccc}
\hline \multirow{2}{*}{ Participantes } & \multicolumn{1}{c}{ Dimensiones } & \multicolumn{3}{c}{ Nivel de denuncias } \\
\cline { 3 - 5 } Profesionales & $\begin{array}{c}\text { Total de denuncias } \\
\text { maltrato } y \\
\text { discriminación }\end{array}$ & $\begin{array}{c}\text { Total de } \\
\text { denuncias por } \\
\text { maltrato escolar }\end{array}$ & $\begin{array}{c}\text { Total de denuncias } \\
\text { por discriminación }\end{array}$ \\
\cline { 3 - 5 } & $\begin{array}{l}\text { Prácticas de } \\
\text { convivencia } \\
\text { inclusiva }\end{array}$ & .073 & .027 & .070 \\
Padres & $\begin{array}{l}\text { Prácticas punitivas } \\
\text { Prácticas de }\end{array}$ & .054 & -.178 & .050 \\
& $\begin{array}{l}\text { convivencia } \\
\text { inclusiva } \\
\text { Prácticas punitivas }\end{array}$ & $-.254^{*}$ & -.098 & $-.234^{*}$ \\
\hline
\end{tabular}

Nota: La correlación es significativa al nivel 0.01 (bilateral)

Elaboración propia, 2017.

\section{Discusión}

El presente estudio buscó construir y validar un instrumento que evaluara la convivencia escolar en Chile desde la perspectiva de las prácticas y el ofrecimiento de múltiples dimensiones para ser valorizado por personas adultas de las escuelas. El propósito fue contribuir a la toma de decisiones informada en esta área. Es importante mencionar que ambas versiones del instrumento revelan acciones concretas que realizan los profesionales al interior de las escuelas, alejándose de la evaluación de las percepciones o evaluaciones de calidad que generalmente se evalúan bajo el constructo de clima escolar o percepción de la convivencia escolar. Además, el instrumento toma como referencia e inspiración la literatura anglosajona y latinoamericana sobre el mejoramiento de la convivencia escolar, la que sostenidamente ha mostrado que es necesario mejorar las prácticas concretas de 
gestión (Bellei, Morawietz, Valenzuela y Vanni, 2017; Bellei, Valenzuela, Vanni y Contreras, 2014).

Los resultados de la validación psicométrica indican que los niveles de consistencia interna son adecuados, con alphas de Cronbach sobre .70 y sobre .80 para las dimensiones de ambos instrumentos (a excepción de la dimensión Prácticas punitivas para profesionales y las dimensiones de Actitudes de disposición al cambio). Para ambos instrumentos, mejoraron los indicadores de ajustes del análisis factorial confirmatorio. Sin embargo, los valores de ajuste no alcanzan a ser adecuados para una dimensión de 2do orden para ambos instrumentos. Además, se realizó un análisis de la validez de criterio mediante correlaciones entre las prácticas de convivencia escolar y el nivel de denuncias por maltrato y discriminación al interior de las escuelas. Los resultados sugieren que, a mayor número de denuncias recibidas por las escuelas, menor es el nivel de frecuencia de prácticas de convivencia escolar inclusiva percibidas por padres y madres al interior de dichas instalaciones. Es necesario señalar que las denuncias las realizan, en su gran mayoría, los propios padres o madres en el sitio web o en las dependencias de la Superintendencia de Educación Escolar. Por lo tanto, estos resultados son coherentes si consideramos la relación entre lo que hace la escuela, según las familias, y las razones que llevan a estas últimas a poner una denuncia.

En conclusión, y teniendo en cuenta cada una de las dimensiones de ambas versiones del instrumento, los resultados cumplen con los criterios psicométricos de validez y confiabilidad. Contar con un instrumento que reporte prácticas concretas inspiradas en la disposición al cambio organizacional para mejorar la convivencia escolar resulta un hito importante para la gestión de la convivencia escolar al interior de las escuelas chilenas, pues permite reconocer e impulsar una gestión formativa y pedagógica que, según la literatura especializada, se relaciona con un mejoramiento sostenido en el tiempo. Asimismo, la diversidad de dimensiones que ofrece este instrumento facilita la identificación de aquellas áreas débiles o fuertes al interior de las instituciones escolares. Esto posibilita precisar respuestas situadas a las diversas realidades escolares. Por último, contar con una versión para profesionales y otra para la familia proporciona una oportunidad para conocer las diversas voces de los actores, lo que, sin duda, beneficia la participación de toda la comunidad escolar.

Sin embargo, el carácter dinámico y situado de las prácticas (Gherardi, 2009) hace poco pertinente sostener que estas puedan normalizarse y estabilizarse en el tiempo. Como 
señala Gherardi (2012), las prácticas proporcionan formas de hacer que se sustentan tanto en las relaciones materiales como sociales. Sin embargo, simultáneamente, este principio ordenador es también temporal e inestable, y por lo tanto es también un principio desorganizador. Por ello, no se recomienda la generación de normas estadísticas o baremos derivados de este o futuros estudios. Por el contrario, en este estudio se sostiene que este instrumento puede utilizarse a modo de linterna, para iluminar ciertas prácticas organizacionales que sostienen ciertos órdenes y principios organizadores de la gestión de la convivencia escolar, y cuya toma de conciencia, por parte de las comunidades escolares, puede ayudar a darse cuenta cuán preparada está una organización escolar para mejorar la convivencia, y qué necesita aprender de sí misma. Ahora bien, atendiendo a lo anterior, quien desee utilizar estos instrumentos haciendo uso de sus baremos o puntos de corte, puede guiarse por los promedios y desviaciones estándar presentados en la Tabla 4. Conforme señala la literatura tradicional, un percentil en o bajo el percentil 25 puede considerarse un nivel bajo; en o cerca del percentil 50, un nivel medio; y en o sobre el percentil 75, un nivel alto (Valero, 2013).

En síntesis, se considera que este instrumento puede ser útil para evaluar la disposición al mejoramiento de la convivencia escolar. Como señalan Tyre et al. (2012), ante la complejidad de las políticas de los modelos de escuela total para satisfacer las necesidades de toda la niñez de las escuelas, los esfuerzos de estas pueden ser infructuosos si no se consideran las características particulares de una escuela como sistema, antes de la implementación. Como consecuencia, es necesario que las escuelas evalúen cuán preparadas y cuán dispuestas están para la implementación inicial de las políticas de mejoramiento del clima escolar. En el contexto creciente de leyes y políticas para la disminución de la violencia escolar y el mejoramiento de la convivencia escolar en diversos países de América Latina, contar con herramientas válidas y confiables para evaluar la disposición y preparación para el cambio, es deseable.

La principal limitación radica en que el instrumento solo fue contestado por adultos/as, por lo tanto, no se cuenta con las percepciones de los estudiantes sobre las prácticas de convivencia escolar reportadas en las diferentes dimensiones. Esto implica que la producción y el posterior análisis de información se realizaron con las percepciones de unos participantes en específico, sin considerar a la población estudiantil. La literatura sobre convivencia escolar es clara en la importancia de los estudiantes como agentes claves de la democracia al interior de la escuela. Por consiguiente, lo que digan y reporten va a ser 
relevante para la toma de decisiones de un centro escolar. De esta manera, el desafío frente a futuras investigaciones es comprobar la pertinencia del instrumento en estudiantes y en otros niveles de enseñanza.

\section{Agradecimientos}

Este estudio fue financiado por el proyecto FONDECYT 1140960 Políticas y prácticas de apoyo a la convivencia escolar en ambientes punitivos, segregados y de pruebas estandarizadas con altas consecuencias: El caso de Chile, y por el proyecto PIA CONICYT CIE 160009.

\section{Referencias}

American School Counselor Association. (2003). The ASCA national model: A framework for school counseling programs. Arlington, Virginia, Estados Unidos: ASCA.

Armenakis, Achiles A., Harris, Stanley G., y Feild, Hubert S. (2000). Making change permanent a model for institutionalizing change interventions. En Richard Woodman, William Pasmore y Abraham Shani (Eds.), Research in organizational change and development (pp. 97-128). Bingley, Reino Unido: Emerald Group Publishing Limited.

Bellei, Cristian, Morawietz, Liliana, Valenzuela, Juan Pablo y Vanni, Xavier. (2017). Nadie dijo que era fácil. Escuelas efectivas en sectores de pobreza, diez años después. Santiago, Chile: LOM Ediciones.

Bellei, Cristián, Vanni, Xavier, Valenzuela, Juan Pablo y Contreras, David (2015). Una mirada a los procesos de mejoramiento escolar en Chile. Apuntes sobre mejoramiento escolar, 2. 1-12.

Bellei, Cristian, Valenzuela, Juan Pablo, Vanni, Xavier y Contreras, Daniel. (Coords.). (2014). Lo aprendí en la escuela ¿Cómo se logran procesos de mejoramiento escolar? Santiago, Chile: LOM Ediciones.

Benbenishty, Rami, Astor, Ron Avi, Roziner, Ilan y Wrabel, Stephani L. (2016). Testing the Causal Links Between School Climate, School Violence, and School Academic Performance: A Cross-Lagged Panel Autoregressive Model. Educational Researcher, 45(3), 197-206. doi: https://doi.org/10.3102/0013189X16644603

Booth, Tony y Ainscow, Mel (2015). Guía para la Educación Inclusiva. Desarrollando el aprendizaje y la participación en los centros escolares [Adaptación de la $3^{\text {a }}$ edición revisada del Index for Inclusion]. Madrid, España: OEI.

Byrne, Bárbara (2010). Structural equation modeling with AMOS, (2 ed.). New York: Routledge. 
Carey, John, Harrity, Jim y Dimmitt, Carey. (2005). The Development of a Self-Assessment Instrument to Measure a School District's Readiness to Implement the ASCA National Model. Professional School Counseling, 8(4), 305-312.

Chaparro Caso-López, Alicia A.; Caso Niebla, Joaquín, Fierro Evans, María Cecilia y Díaz López, Carlos. (2015). Desarrollo de un instrumento de evaluación basado en indicadores de convivencia escolar democrática, inclusiva y pacífica. Perfiles Educativos, 37(149), 20-41. $\quad$ Recuperado de http://www.redalyc.org/articulo.oa?id=13239889002

Cohen, Jonathan, McCabe, Libby, Michelli, Nicholas M. y Pickeral, Terry. (2009). School climate: Research, Policy, Practice and Teacher Education. Teachers College Record, 111(1), 180-213.

Cohen, Jonathan, Espelage, Dorothy L., Twemlow, Stuart, Berkowitz, Marvin W. y Comer, James P. (2015). Rethinking Effective Bully and Violence Prevention Efforts: Promoting Healthy School Climates, Positive Youth Development, and Preventing Bully-VictimBystander Behavior. International Journal of Violence and Schools, 15(1), 2-40.

Cohen, Jonathan and Thapa, Amrit. (2017). School Climate Improvement: What Do U.S. Educators Believe, Need and Want?. International Journal on School Climate and Violence Prevention, 2(1), 90-116.

Creemers, Bert P. M., y Leonidas, Kyriakides. (2010). Using the Dynamic Model to develop an evidence-based and theory-driven approach to school improvement. Irish Educational Studies, 29(1), 5-23. doi: 10.1080/03323310903522669

Dary, Teri y Pickeral Terry. (2013). School Climate Practices for Implementation and Sustainability. School Climate Practice Briefs, 1, 1-56. Recuperado de http://www.ijvs.org/files/Publications/School-Climate-Practice-Briefs-for-Implementationand-Sustainability-2013.pdf

De Pedro, Kris Tunac, Pineda, Diana, Capp, Gordon, Moore, Hadass, Benbenishty, Rami y Astor, Ron Avi. (2017). Implementation of a School Districtwide Grassroots Antibullying Initiative: A School Staff and Parent-Focused Evaluation of Because Nice Matters. Children y Schools, 39(3), 137-145. doi: https://doi.org/10.1093/cs/cdx008

Dimmit C. y Robillard, L. (2014). Evidence-Based Practices: Pro-social skill development and violence prevention in K-8 Schools [Documento inédito]. Massachusets, Estados Unidos: Fredrickson Center for School Counseling Outcome Research and Evaluation, UMass Amherst.

Durán Rodríguez, María Martha. (2016). Bullying: políticas de atención y prevención en Costa Rica. Revista Posgrado y Sociedad, 14(1), 25-38.

Elfrink, Teuntje R., Goldberg, Jochem M., Schreurs, Karlein M. G., Bohlmeijer, Ernst T. and Clarke, Aleisha M. (2017). Positive educative programme: A whole school approach to supporting children's well-being and creating a positive school climate: a pilot study. Health Education, 117(2), 215-230. doi: https://doi.org/10.1108/HE-09-2016-0039 
Elmore, Richard y City, Elizabeth. (2007). The Road to School Improvement: It's hard, it's bumpy, and it takes as long as it takes. Harvard Education Letter, 23(3). Recuperado de http://hepg.org/hel-home/issues/23 3/helarticle/the-road-to-schoolimprovement 229

Fardella, Carla y Carvajal, Francisca. (2018). Los estudios sociales de la práctica y la práctica como unidad de estudio. Psicoperspectivas, 17(1). https://doi.org/10.5027/psicoperspectivas-vol17-issue1-fulltext-1241

Fierro Evans, María Cecilia. (2013). Convivencia inclusiva y democrática: Una perspectiva para gestionar la seguridad escolar. Revista Electrónica Sinéctica, (40), 1-18. Recuperado de http://www.redalyc.org/articulo.oa?id=99827467006

Gairín, Joaquín y Barrera-Corominas, Aleix. (2014). La convivencia en los centros educativos de educación básica en Iberoamérica. Santiago, Chile: Editorial EDP-UAB.

Gálvez Nieto, José Luis, Tereucan Angulo, Julio César, Muñoz Navarro, Sergio, Briceño Olivera, Claudio Andrés y Mayorga Muñoz, Cecilia. (2014). Propiedades psicométricas del cuestionario para evaluar clima social del centro escolar (CECSCE). Liberabit, 20(1), 165-174. Recuperado de http://132.248.9.34/hevila/Liberabit/2014/vol20/no1/13.pdf

Gatica, Miguel (2017). Violencia escolar en Chile. Una aproximación no criminalizada (tesis doctoral). Universitat abat oliba ceu, Barcelona, España.

Gherardi, Silvia. (2009). Organizational Knowledge: The Texture of Workplace Learning. Hoboken, NJ: John Wiley.

Gherardi, Silvia. (2012). How to Conduct a Practice-Based Study Problems and methods. Cheltenham, Reino Unido: Edward Elgar Publishing.

Gray, J., Hopkins. D., Reynolds, D., Wilcox, B., Farrel, S. y Jesson, D. (1999). Improving Schools: Performance and Potential. Buckingham, Reino Unido: Open University Press.

Hallinger, Phillip y Heck, Ronald (2011). Exploring the journey of school improvement: Classifying and analyzing patterns of change in school improvement processes and learning outcomes. School Effectiveness and School Improvement, 22(1), 1-27.

Helfrich, Christian D., Li, Yu-Fang, Sharp, Nancy D. y Sales, Anne E. (2009). Organizational readiness to change assessment (ORCA): Development of an instrument based on the Promoting Action on Research in Health Services (PARIHS) framework. Implementation Science, 4(38). doi: https://doi.org/110.1186/1748-5908-4-38

Hernández, Roberto, Fernández, Carlos y Baptista, Pilar. (2010). Metodología de Investigación (5ª . ed.). D.F., México: Mc Graw Hill.

Kazak, Anne E., Hoagwood, Kimberly, Weisz, John R., Hood, Korey, Kratochwill, Thomas R., Vargas, Luis A. y Banez, Gerard A. (2010). A Meta-Systems Approach to EvidenceBased Practice for Children and Adolescents. American Psychologist, 65(2), 85-97. doi: https://doi.org/10.1037/a0017784 
Ley del Sistema Nacional de Convivencia Escolar y Formación para el Ejercicio de los Derechos Humanos, la Educación para la Sexualidad y la Prevención y Mitigación de la Violencia Escolar, № 1620. (2013). Recuperado de https://www.mineducacion.gov.co

Ley que promueve la convivencia sin violencia en las instituciones educativas, $\mathrm{N}^{\circ} 29.719$. (2011).

Recuperado

de http://www2.congreso.gob.pe/sicr/cendocbib/con4 uibd.nsf/36F4D51A64BB872805257 9F90061B160/\$FILE/1 LEY 29719.pdf

Ley para la promoción de la convivencia y el abordaje de la conflictividad social en las instituciones educativas, $\mathrm{N}^{\circ}$ 26.892. (2013). Recuperado de http://cdh.defensoria.org.ar

Ley de Violencia escolar, N²0.536. (2011). Recuperado de https://www.leychile.cl

López, Verónica, Carrasco, Claudia, Morales, Macarena, Ayala, Alvaro, López, Joedith y Karmy, Michelle. (2011). Individualizando la violencia escolar: Análisis de prácticas discursivas en una escuela de la Región de Valparaíso. Psykhe, 20(2), 75-91.

López, Verónica, Bilbao, María Ángeles, Ascorra, Paula, Moya, Iván y Morales, Macarena. (2014). Escala de Clima Escolar: adaptación al español y validación en estudiantes chilenos. Universitas Psychologica, 13(3), 1111-1121. doi: https://doi.org/110.11144/Javeriana.UPSY13-3.ecea

Méndez Martínez, Carolina y Rondón Sepúlveda, Martín Alonso. (2012). Introducción al análisis factorial exploratorio. Revista Colombiana de Psiquiatría, 41(1), 97-207. Recuperado de http://www.redalyc.org/articulo.oa?id=8062409301.

National School Climate Center. (2014). School Climate Practice. Recuperado de https://www.schoolclimate.org/publications/practice

Pérez, Francisco, Rodríguez, Juan, Romero, Bélgica, Ruvalcaba, Francisco y Lozano, Oscar. (2002). Preferencias por formatos de respuesta en cuestionarios para encuestas. Metodología de encuestas, 4(1), 63-74.

Prochaska, Janice, Prochaska, James, y Levesque, Deborah. (2001). A transtheoretical approach to changing organizations. Administration and Policy in Mental Health, 28(4), 247-261.doi: http://dx.doi.org/10.1023/A:1011155212811

Programa de las Naciones Unidas para el Desarrollo. (2009). Informe de desarrollo humano en Chile: La manera de hacer las cosas. Santiago, Chile: Autor.

Sandoval Márquez, Mario. (2014). Convivencia y clima escolar: claves de la gestión del conocimiento. Última Década, 22(41), 153-178. doi: https://dx.doi.org/10.4067/S0718$\underline{22362014000200007}$

Senge, Peter, Cambron-McCabe, Nelda, Lucas, Timothy, Smith, Bryan, Dutton, Janis, y Kleiner, Art. (2000). Schools that learn. Nueva York, Estados Unidos: Doubleday. 
Speece, Deborah L., Case, Lisa Pericola y Molloy, Dawn Eddy. (2003). Responsiveness to General Education Instruction as the First Gate to Learning Disabilities Identification. Learning Disabilities Research \& Practice, 18(3), 147-156. doi: https://doi.org/10.1111/1540-5826.00071

Stevens, Gregory W. (2013). Toward a process-based approach of conceptualizing change readiness. The Journal of Applied Behavioral Science, 49(3). doi: https://doi.org/10.1177/0021886313475479

Thomas, Felicity and Aggleton, Peter. (2016). A confluence of evidence What lies behind a "whole school" approach to health education in schools?. Health Education, 116(2). doi: https://doi.org/10.1108/HE-10-2014-0091

Tyre, Ashli D., Feuerborn, Laura, Beisse, Kay and McCready, Courtney. (2012). Creating readiness for response to intervention: An evaluation of readiness assessment tools. Contemporary School Psychology, 16(1), 103-114.

Valdés, René, López, Verónica y Chaparro, Alicia. (en prensa). Convivencia escolar: adaptación y validación de un instrumento mexicano en Chile. Revista Electrónica de Investigación Educativa, 20(4).

Valero, Sergi. (2013). Transformación e interpretación de las puntuaciones. En Julio Meneses (Ed), Psicometría (pp. 201-224). Barcelona, España: editorial UOC.

Valenzuela, Jaime, Ahumada, Iván, Rubilar, Andrea, López, Verónica y Urbina, Carolina. (2018). El encargado de convivencia escolar en Chile: Hacia la comprensión de su identidad laboral. Revista de Psicología, 36(1), 189-216. doi: https://doi.org/10.18800/psico.201801.007

Villaoslada, Emiliana y Torrego Seijo, Juan Carlos. (2004). Modelo integrado de regulación de la convivencia y tratamiento de conflictos. Revista Pedagógica, 18, 31-48. Recuperado de https://dialnet.unirioja.es/servlet/articulo?codigo=1138351

Wang, Ming Te y Degol, Jessica L. (2016). School Climate: a Review of the Construct, Measurement, and Impact on Student Outcomes. Educational Psychology Review, 28(2), 315-352. doi: https://doi.org/10.1007/s10648-015-9319-1

Weiner, Bryan, Amick, Halle y Lee, Shoou. (2008). Review: Conceptualization and measurement of organizational readiness for change. A review of the literature in health services research and other fields. Medical Care Research and Review, 65(4), 379-436. doi: $10.1177 / 1077558708317802$

Wilson, David B., Gottfredson, Denise C. y Najaka, Stacy S. (2001). School-Based Prevention of Problem Behaviors: A Meta-Analysis. Journal of Quantitative Criminology, 17(3), 247-272. 
Anexo 1. Instrumento final para profesionales y padres

\begin{tabular}{|c|c|c|}
\hline Dimensiones & Reactivos & $\begin{array}{l}\text { Versión } \\
\text { final }\end{array}$ \\
\hline \multirow[t]{8}{*}{ Cultura inclusiva } & $\begin{array}{l}\text { Existe un ambiente familiar/Existe un ambiente familiar y } \\
\text { acogedor. }\end{array}$ & $\checkmark$ \\
\hline & $\begin{array}{l}\text { Nos sentimos orgullosos de trabajar aquí/Nos sentimos } \\
\text { orgullosos de participar de esta comunidad escolar. }\end{array}$ & $\checkmark$ \\
\hline & $\begin{array}{l}\text { Cuando llega un nuevo profesor, recibe una inducción } \\
\text { (solo profesionales) }\end{array}$ & $\checkmark$ \\
\hline & $\begin{array}{l}\text { Existe confianza y buena comunicación entre todos los } \\
\text { actores de la comunidad escolar. }\end{array}$ & $\checkmark$ \\
\hline & Los padres y apoderados confían en la escuela. & $\checkmark$ \\
\hline & $\begin{array}{l}\text { Existe una comunicación fluida con los padres y } \\
\text { apoderados }\end{array}$ & $\checkmark$ \\
\hline & $\begin{array}{l}\text { Percibimos a los estudiantes contentos y a las familias } \\
\text { satisfechas. }\end{array}$ & $\checkmark$ \\
\hline & $\begin{array}{l}\text { Hay muchas tradiciones que nos hacen sentir parte de } \\
\text { ella. }\end{array}$ & $\checkmark$ \\
\hline \multirow[t]{6}{*}{ Prácticas inclusivas } & $\begin{array}{l}\text { Existen estrategias efectivas para potenciar a los } \\
\text { estudiantes con intereses diversos. }\end{array}$ & $\checkmark$ \\
\hline & $\begin{array}{l}\text { Se identifican a tiempo a los estudiantes que presentan } \\
\text { necesidades de apoyo (sociales, afectivas y } \\
\text { conductuales) }\end{array}$ & $\checkmark$ \\
\hline & $\begin{array}{l}\text { Existen mecanismos efectivos para apoyar a los } \\
\text { estudiantes que presentan problemas de aprendizaje. }\end{array}$ & $\checkmark$ \\
\hline & $\begin{array}{l}\text { Existen mecanismos efectivos para apoyar a los } \\
\text { estudiantes que presentan problemas de conducta. }\end{array}$ & $\checkmark$ \\
\hline & $\begin{array}{l}\text { Los estudiantes más aventajados ayudan a los que } \\
\text { presentan barreras para el aprendizaje/Los estudiantes } \\
\text { más aventajados ayudan a los que presentan problemas } \\
\text { de aprendizaje. }\end{array}$ & $\checkmark$ \\
\hline & $\begin{array}{l}\text { Los profesores monitorean constantemente el aprendizaje } \\
\text { de los estudiantes/Los profesores supervisan } \\
\text { constantemente el aprendizaje de los estudiantes. }\end{array}$ & $\checkmark$ \\
\hline \multirow[t]{8}{*}{ Formación continua } & $\begin{array}{l}\text { Al equipo directivo le interesa el desarrollo y la formación } \\
\text { continua de los profesores/Al equipo directivo le interesa } \\
\text { el desarrollo y la capacitación constante de los } \\
\text { profesores. }\end{array}$ & $\checkmark$ \\
\hline & $\begin{array}{l}\text { Se promueve el perfeccionamiento docente de todos los } \\
\text { profesores/Se promueve que los profesores sigan } \\
\text { estudiando y se perfeccionen. }\end{array}$ & $\checkmark$ \\
\hline & Existe una cultura de mejoramiento continuo. & $\checkmark$ \\
\hline & $\begin{array}{l}\text { Los profesores y demás profesionales pueden proponer } \\
\text { cosas nuevas (profesionales) }\end{array}$ & $\checkmark$ \\
\hline & $\begin{array}{l}\text { Son acogidas las propuestas formuladas por los } \\
\text { profesores y demás profesionales (profesionales) }\end{array}$ & $\checkmark$ \\
\hline & $\begin{array}{l}\text { La formación continua y el crecimiento profesional están } \\
\text { altamente arraigados en la cultura escolar. }\end{array}$ & $\checkmark$ \\
\hline & $\begin{array}{l}\text { Se promueve la formación de profesionales no docentes } \\
\text { (psicólogos, asistentes sociales, etc)/Se promueve la } \\
\text { capacitación de profesionales no docentes (psicólogos, } \\
\text { asistentes sociales, etc). }\end{array}$ & $\checkmark$ \\
\hline & $\begin{array}{l}\text { Siempre estamos evaluando lo que hacemos y nos } \\
\text { retroalimentamos. }\end{array}$ & $\checkmark$ \\
\hline \multirow[t]{4}{*}{ Orientación al trabajo en equipo } & $\begin{array}{l}\text { Todos tenemos claros nuestros objetivos como miembros } \\
\text { de esta comunidad escolar. }\end{array}$ & $\checkmark$ \\
\hline & $\begin{array}{l}\text { Todos trabajamos para llevar a cabo objetivos comunes/ } \\
\text { Todos colaboramos para llevar a cabo objetivos comunes. }\end{array}$ & $\checkmark$ \\
\hline & $\begin{array}{l}\text { Los profesores comparten sus buenas prácticas para } \\
\text { mejorar la convivencia escolar. }\end{array}$ & $\checkmark$ \\
\hline & Los profesores comparten sus buenas prácticas para & $\checkmark$ \\
\hline
\end{tabular}


Normas compartidas

Participación democrática

Prácticas punitivas

Actitudes de disposición al cambio

Disposición organizacional mejorar los aprendizajes.

Existe una responsabilidad compartida: los triunfos y fracasos son de todos.

Las normas son claras y conocidas por todos/as.

Las normas se aplican con justicia

Los profesores se apoyan en el reglamento escolar para resolver los problemas que se presentan en la sala de clases.

Los asistentes de la educación se apoyan en el reglamento escolar para resolver los problemas que se presentan fuera del aula.

El equipo directivo se apoya en el reglamento escolar para resolver los problemas que se presentan en la escuela.

Los estudiantes están motivados y participan activamente en la vida de la escuela.

Se atiende a los padres y apoderados que requieren atención especial para sus hijos.

Se respetan las ideas de los estudiantes.

Se les motiva a los estudiantes para que puedan expresar sus ideas.

Se toman en cuenta las opiniones de los padres y apoderados sobre la formación de sus hijos

Se toman en cuenta las opiniones de los estudiantes respecto de su formación.

Se crean las instancias para que participen los vecinos $u$ otros miembros de la comuna

Los vecinos del barrio tiene una opinión positiva de la escuela.

Los padres y apoderados participan activamente en las soluciones antes los problemas de convivencia escolar.

Los padres y apoderados participan del diseño de protocolos y reglamentos de convivencia escolar.

Los estudiantes participan del diseño de protocolos y reglamentos de convivencia escolar.

Se castiga a los estudiantes que presentan problemas de conducta.

Se suspende temporalmente de la escuela a los estudiantes que cometen faltas.

Se expulsa de la sala de clases a los estudiantes que interfieren el buen clima de aula.

A fin de año se invita a los estudiantes con problemas de conducta a buscar otro ambiente educativo

Se deja sin recreo a los estudiantes que no terminan sus tareas durante la clase.

Se envía a inspectoría a los estudiantes que presentan mayores problemas de comportamiento/ Se envía a inspectoría a los estudiantes que presentan mayores problemas de comportamiento

Creemos que es necesario incorporar cambios para mejorar la convivencia escolar.

Existe una apertura constante al cambio para el desarrollo de esta escuela.

El equipo directivo apoya los cambios propuestos para mejorar la convivencia escolar.

Existe una disposición constante al cambio cuando las estrategias no dan resultados.

Los profesores y profesionales de apoyo conforman un equipo de trabajo cohesionado/Los profesores y profesionales de apoyo conforman un equipo de trabajo unido y responsable. 
Cultura de mejoramiento de la convivencia

Prevención de los problemas de convivencia

Promoción de la buena convivencia
Los profesores y profesionales de apoyo conforman un equipo de trabajo competente/Los profesores y profesionales de apoyo conforman un equipo de trabajo bien preparado.

Los profesores en general tienen una buena opinión de sus colegas.

Existe una buena comunicación entre profesores y el equipo directivo.

El equipo directivo comunica a los miembros de la comunidad escolar que siempre pueden mejorar sus prácticas.

Las reuniones periódicas sí sirven para resolver los problemas y tomar decisiones (profesionales)

El equipo directivo, profesores y profesionales no docentes comparten la misma visión sobre convivencia escolar (profesionales)

Han habido acciones para la mejora de la convivencia escolar, que demuestran el compromiso de la escuela sobre este tema.

Se incluye a todos los miembros de la comunidad (estudiantes, padres y apoderados, etc) en el proceso de la mejora de la convivencia escolar.

Uno de los objetivos explícitos es el desarrollo de una cultura de la confianza.

Uno de los objetivos de esta escuela es el fomento de la solidaridad.

Existen los recursos necesarios para apoyar los procesos de mejoramiento de la convivencia escolar.

El equipo directivo señala que el mejoramiento de la convivencia escolar es un proceso continuo y tarea de todos.

La reflexión es una habilidad explícita (se habla de ella) y es bien valorada por la comunidad.

Se adoptan medidas preventivas para evitar los problemas de convivencia.

Se identifican a tiempo juegos violentos en el recreo.

Se identifican a tiempo los conflictos entre estudiantes y profesores

Se identifican a tiempo las relaciones violentas entre estudiantes (sean de compañeros, pololeo o amistad).

Se utilizan los consejos de cursos para tratar temas que mejoren la convivencia.

Se enseña la importancia del respeto entre compañeros y de tratar a todos y a todas por igual/ Se enseña la importancia del respeto entre compañeros y de tratar a todos y a todas por igual.

Se promueve la resolución pacífica de conflictos.

Se promueve la tolerancia, el compañerismo y la solidaridad entre los miembros del establecimiento.

Se trabajan cotidianamente los objetivos fundamentales transversales del currículum.

Existen instancias y actividades de fortalecimiento para el aprendizaje de habilidades emociones y sociales.

Se les invita a los estudiantes a reflexionar sobre las consecuencias de la violencia escolar/ Se les invita a los estudiantes a reflexionar sobre las consecuencias de la violencia escolar.

Nota: En el caso de los ítems separados por un slash (/), los de la izquierda corresponden a profesionales y los de la derecha a padres. Lo mismo ocurre con los símbolos de las casillas. Fuente: Elaboración propia. 